BULL. AUSTRAL. MATH. SOC.

VOL. 31 (1985), 245-255.

\title{
ON THE ZEROS OF A POLYNOMIAL AND ITS DERIVATIVE
}

\author{
ABDUL AZIZ
}

Let $P(z)$ be a polynomial of degree $n$ and $P^{\prime}(z)$ be its derivative. Given a zero of $P^{\prime}(z)$, we shall determine regions which contains at least one zero of $P(z)$. In particular, it will be shown that if all the zeros of $P(z)$ lie in $|z| \leq 1$ and $w_{1}, w_{2}, \ldots, w_{n-1}$ are the zeros of $P^{\prime}(z)$, then each of the disks $\left|(z / 2)-w_{j}\right| \leq \frac{1}{2}$ and $\left|z-w_{j}\right| \leq 1, j=1,2, \ldots, n-1$, contains at least one zero of $P(z)$. We shall also determine regions which contain at least one zero of the polynomials $m P(z)+z P^{\prime}(z)$ and $P^{\prime}(z)$ under some appropriate assumptions. Finally some other results of similar nature will be obtained.

\section{Introduction and statement of results}

Let all the zeros of a polynomial $P(z)$ of degree $n$ lie in the closed unit disk $|z| \leq 1$ and let $P(a)=0$, then according to a conjecture of Sendov, better known as "Ilieff's conjecture" [4, Problem 4.5], [6, p. 795], the disk $|z-a| \leq 1$ contains at least one zero of $P^{\prime}(z)$, the derivative of $P(z)$. The boundary case, that is when $|a|=1$, has been proved by Rubinstein [10]. A conjecture stronger than that of Ilieff, in which the disk $|z-a| \leq 1$ is replaced by the disk $|z-(a / 2)| \leq 1-|a| / 2$, is stated in [3] by Goodman, Rahman and Ratti and

Received 5 October 1984.

Copyright Clearance Centre, Inc. Serial-fee code: 0004-9727/85 $\$$ A2.00 + 0.00 . 
is proved there only for the boundary case. In full generality, these conjectured results have been proved [2], [3], [6], [7], [10], [12] only for polynomials of degree at most five.

Ilieff's conjecture might suggest that close to every zero of $P(z)$ there should always lie a zero of $P^{\prime}(z)$. In this paper we shall first determine a neighbourhood of a zero $w$ of $P^{\prime}(z)$, which will always contain a zero of $P(z)$. We prove

THEOREM 1. If $P(z)$ is a polynomial of degree $n$ and $w$ is a zero of $P^{\prime}(z)$, then for every given real or complex number $\alpha, P(z)$ has at least one zero in the region

$$
\left|w-\frac{\alpha+z}{2}\right| \leq\left|\frac{\alpha-z}{2}\right|
$$

Taking $\alpha=0$ in Theorem $I$ and noting that $|w-(z / 2)| \leq|z / 2|$ implies $|\omega-z| \leq|z|$, we get the following interesting result.

COROLLARY 1. If all the zeros of a polynomial $P(z)$ of degree $n$ lie in $|z| \leq 1$, and $w$ is a zero of $P^{\prime}(z)$, then $P(z)$ has at least one zero in both the circles

$$
\left|w-\frac{z}{2}\right| \leq \frac{1}{2} \text { and }|w-z| \leq 1 \text {. }
$$

Next we prove

THEOREM 2. If all the zeros of a polynomial $P(z)$ of degree $n$ lie in $|z| \leq 1$ and $P(a)=0, a \neq 0$, then for every positive integer $m$, the polynomial $F(z)=m P(z)+z P^{\prime}(z)$ has at least one zero in the circle

$$
|z-a| \leq 1 \text {. }
$$

THEOREM 3. If $P(z)=(z-a) Q(z)$ is a polynomial of degree $n$ and if all the zeros of $Q(z)$ lie in the circle $|z+\alpha-a| \leq|\alpha|$ for some real or complex number $a \neq 0$, then at least one zero of $P^{\prime}(z)$ lies in the circle

$$
\left|z-a+\frac{\alpha}{2}\right| \leq\left|\frac{\alpha}{2}\right| .
$$

For $a=I=\alpha$, this reduces to the result of Goodman, Rahman and Ratti [3].

An immediate consequence of Theorem 3 is 
COROLLARY 2. If all the zeros of a polynomial $P(z)=(z-a) Q(z)$, $0 \leq a \leq 1$, lie in the region $S=\{|z| \leq 1\} \cap\{|z+1-a| \leq 1\}$, then $P^{\prime}(z)$ has at least one zero in both the circles

$$
\left|z-a+\frac{1}{2}\right| \leq \frac{1}{2} \text { and }\left|z-\frac{a}{2}\right| \leq 1-\frac{a}{2} \text {. }
$$

THEOREM 4. Let $P(z)=(z-a) Q(z)$ be a polynomial of degree $n$, If

$$
\operatorname{Re} \frac{\alpha Q^{\prime}(a)}{(n-1) Q(a)} \geq \frac{3}{2},
$$

for some real or complex number $\alpha$, then $P^{\prime}(z)$ has at least one zero in the circle

$$
\left|z-\alpha+\frac{\alpha}{2}\right| \leq\left|\frac{\alpha}{2}\right|
$$

The next corollary immediately follows from Theorem 4.

COROLLARY 3. If $P(z)=(z-1) Q(z)$ is a polynomial of degree $n$ and $P^{\prime}(z)$ does not vanish in the circle $\left|z-\frac{1}{2}\right| \leq \frac{1}{2}$, then

$$
\operatorname{Re} \frac{Q^{\prime}(1)}{Q(1)}<\frac{n-1}{2} \text {. }
$$

We also prove

THEOREM 5. If the polynomial $P(z)=(z-1) Q(z)$ of degree $n$ has all its zeros in $|z| \geq 1$, then $P^{\prime}(z)$ cannot have all its zeros in the disk

$$
\left|z-\frac{1}{2}\right|<\frac{1}{2} \text {. }
$$

Finally we establish

THEOREM 6. If $P(z)$ is a polynomial of degree $n$ such that

$$
\operatorname{Max}_{|z|=1}|P(z)|=\left|P\left(e^{i \theta}\right)\right|,
$$

then $P(z)$ cannot have all its zeros in the disk

$$
\left|z-\frac{e^{i \theta}}{2}\right|<\frac{1}{2} \text {. }
$$

\section{Proofs}

For the proofs of these theorems we need the following lemmas. 
LEMMA 1. If $P(z)$ is a polynomial of degree $n$ such that $P(a)=P(b), a \neq b$, then $P^{\prime}(z)$ has at least one zero in each of the regions

$$
|z-a| \leq|z-b| \text { and }|z-a| \geq|z-b| \text {. }
$$

Proof of Lemma 1. Without loss of generality we suppose $P(a)=P(b)=0$. Consider the polynomial

$$
G(z)=P\left(\left(\frac{a-b}{2}\right) z+\frac{a+b}{2}\right),
$$

then $G(1)=P(a)=0$ and $G(-1)=P(b)=0$. Now it follows from the proof of the Grace-Heawood theorem [5, p. 107] that $G^{\prime}(z)$ is apolar to the polynomial

$$
H(z)=\frac{(z-1)^{n}-(z+1)^{n}}{n},
$$

whose zeros are $z_{k}=-i \cot (k \pi / n), k=1,2, \ldots, n-1$. Since all the zeros of $H(z)$ lie in $\operatorname{Re} z \geq 0$, it follows by Grace's theorem [5, p. 61] that $G^{\prime}(z)$ has at least one zero in $\operatorname{Re} z \geq 0$. That is, at least one zero of $G^{\prime}(z)$ lie in $|z-1| \leq|z+1|$. Replacing $z$ by $(z-((a+b) / 2))((2 /(a-b)))$, it follows that, at least one zero of $P^{\prime}(z)$ lies in $|z-a| \leq|z-b|$.

Since all the zeros of $H(z)$ lie also in $\operatorname{Re} z \leq 0$, it follows by a similar argument as above that $P^{\prime}(z)$ has at least one zero in the region $|z-a| \geq|z-b|$. This completes the proof of Lemma 1 .

Let $P(z)$ be a polynomial of degree $n$. The first polar derivative of $P(z)$ with respect to the point $\alpha_{1}$ is defined by

$$
D_{\alpha_{1}} P(z)=n P(z)+\left(\alpha_{1}-z\right) P^{\prime}(z) \text {. }
$$

Similarly the second polar derivative of $P(z)$ with respect to $\alpha_{2}$ is defined by

$$
D_{\alpha_{1}} D_{\alpha_{2}} P(z)=D_{\alpha_{2}}\left(D_{\alpha_{1}} P(z)\right),
$$

and so on. For the proof of Theorem 4, we need

LEMMA 2. If all the zeros of a polynomial $P(z)$ of degree $n$ lie 
in a circular region $C$ and if none of the points $\alpha_{1}, \alpha_{2}, \ldots, \alpha_{k}$, $k \leq n-1$, lies in region $C$, then each of the polar derivatives

$$
D_{\alpha_{1}} P(z), D_{\alpha_{1}} D_{\alpha_{2}} P(z), \ldots, D_{\alpha_{1}} D_{\alpha_{2}} \cdots D_{\alpha_{k}} P(z)
$$

has all of its zeros in region $C$.

This lemma follows by repeated application of Laguerre's theorem [5, p. 49].

Finally for the proof of Theorem 6, we need the following lemma which is an immediate consequence of Berstein's theorem on the derivative of a trigonometric polynomial [11] (see also [1]).

LEMMA 3. Let $P(z)$ be a polynomial of degree $n \geq 1$, then

$$
\operatorname{Max}_{|z|=1}\left|P^{\prime}(z)\right| \leq n \operatorname{Max}_{|z|=1}|P(z)|
$$

\section{Proofs of the theorems}

Proof of Theorem 1. Let $z_{1}, z_{2}, \ldots, z_{n}$ be the zeros of $P(z)$ and let $w$ be a zero of $P^{\prime}(z)$. If $w=\alpha$ or $w=z_{j}$ for some $j=1,2, \ldots, n$, then the result follows and we have nothing to prove. Hence we suppose that $w \neq \alpha$ and $w \neq z_{j}$ for any $j=1,2, \ldots, n$. Since $w$ is a zero of $P^{\prime}(z)$ and $P(w) \neq 0$, we have

$$
\sum_{j=1}^{n} \frac{1}{w-z_{j}}=\frac{P^{\prime}(w)}{P(w)}=0 \text {. }
$$

This gives

$$
\sum_{j=1}^{n} \frac{\left(w-z_{j}\right)-\left(\alpha-z_{j}\right)}{w-z_{j}}=\sum_{j=1}^{n} \frac{w-\alpha}{w-z_{j}}=0
$$

and therefore

$$
\sum_{j=1}^{n} \frac{\alpha-z j}{w-z_{j}}=n
$$

This implies 


$$
n=\sum_{j=1}^{n} \operatorname{Re} \frac{\alpha-z j}{w-z_{j}} \leq n \underset{1 \leq j \leq n}{\operatorname{Max}} \operatorname{Re} \frac{\alpha-z_{j}}{w-z_{j}}
$$

which shows that for at least one $j=1,2, \ldots, n$,

$$
\operatorname{Re} \frac{\alpha-z j}{w-z_{j}} \geq 1
$$

Thus for at least one $j=1,2, \ldots, n$ we have

$$
\left|1-\frac{\alpha-z_{j}}{2\left(w-z_{j}\right)}\right| \leq\left|\frac{\alpha-z_{j}}{2\left(w-z_{j}\right)}\right| .
$$

This gives

$$
\left|w-\frac{\alpha+z j}{2}\right| \leq\left|\frac{\alpha-z j}{2}\right|
$$

for at least one $j=1,2, \ldots, n$, which is equivalent to the desired result.

Proof of Theorem 2. Consider the polynomial

$$
G(z)=z^{m} P(z)
$$

where $m$ is a positive integer greater or equal to 1 , then

$$
G^{\prime}(z)=z^{m-1}\left(m P(z)+z P^{\prime}(z)\right)=z^{m-1} F(z) .
$$

By hypothesis, $P(a)=0, a \neq 0$; therefore $G(a)=0=G(0)$. Hence by using Lemma 1 , with $b=0$, it follows that the polynomial $G^{\prime}(z)$ has at least one zero in the region

$$
|z-a| \leq|z|
$$

As $a \neq 0$, this zero cannot be $z=0$. Therefore this zero must be a zero of $F(z)$. Since all the zeros of $P(z)$ lie in $|z| \leq 1$, it follows by the Gauss-Lucas theorem that all the zeros of $G^{\prime}(z)$ lie in $|z| \leq 1$ and hence all the zeros of $F(z)$ also lie in $|z| \leq 1$. Thus from (1) we conclude that at least one zero of $F(z)$ lie in the circle $|z-a| \leq 1$ and this completes the proof of Theorem 2.

Proof of Theorem 3. We have

$$
P^{\prime}(z)=(z-a) Q^{\prime}(z)+Q(z)
$$


and

$$
P^{\prime \prime}(z)=(z-a) Q^{\prime \prime}(z)+2 Q^{\prime}(z) \text {. }
$$

If $z=a$ is a multiple zero of $P(z)$, then $z=a$ is also a zero of $P^{\prime}(z)$ and since $z=a$ lies in the circle $|z-a+(\alpha / 2)| \leq|\alpha / 2|$, the assertion is true in this case. Henceforth we assume that $z=a$ is a simple zero of $P(z)$, so that $P^{\prime}(a) \neq 0$. Now from (2) and (3) we get

$$
\frac{P^{\prime \prime}(a)}{P^{\prime}(a)}=\frac{2 Q^{\prime}(a)}{Q(a)} \text {. }
$$

If $z_{1}, z_{2}, \ldots, z_{n-1}$ are the zeros of $Q(z)$ and $w_{1}, w_{2}, \ldots, w_{n-1}$ are those of $P^{\prime}(z)$, then from (4) we have

$$
\sum_{j=1}^{n-1} \frac{1}{a-w_{j}}=2 \sum_{j=1}^{n-1} \frac{1}{a-z_{j}} \text {. }
$$

Multiplying the two sides of this equation by $\alpha \neq 0$ and then taking the real parts on both sides, we obtain

$$
\sum_{j=1}^{n-1} \operatorname{Re} \frac{\alpha}{a-w_{j}}=2 \sum_{j=1}^{n-1} \operatorname{Re} \frac{\alpha}{\alpha-\left(\alpha-a+z_{j}\right)} \text {. }
$$

Since by hypothesis

$$
\left|\frac{\alpha-a+z}{\alpha}\right| \leq 1 \text { for all } j=1,2, \ldots, n-1
$$

therefore,

$$
\operatorname{Re} \frac{\alpha}{\alpha-\left(\alpha-a+z_{j}\right)} \geq \frac{1}{2}
$$

for all $j=1,2, \ldots, n-1$. Hence from (5) we get

$$
\sum_{j=1}^{n-1} \operatorname{Re} \frac{\alpha}{a-w_{j}} \geq 2 \sum_{j=1}^{n-1} \frac{1}{2}=n-1 .
$$

This shows that

$$
\text { Re } \frac{\alpha}{a-w_{j}} \geq 1 \text {, for al least one } j=1,2, \ldots, n-1 \text {, }
$$

from which it follows that 


$$
\left|w_{j}-a+\frac{\alpha}{2}\right| \leq\left|\frac{\alpha}{2}\right| \text { for at least one } j=1,2, \ldots, n-1 .
$$

This is equivalent to the desired result and Theorem 3 is proved.

Proof of Theorem 4. If $z=a$ is a multiple zero of $P(z)$, then the result follows as in the proof of Theorem 3. Hence we assume that $z=a$ is a simple zero of $P(z)$, so that $P^{\prime}(a) \neq 0$. Since $P(z)=(z-a) Q(z)$, therefore, $P^{\prime}(a)=Q(a) \neq 0, P^{\prime \prime}(a)=2 Q^{\prime}(a)$. Also it follows by hypothesis that $Q^{\prime}(a) \neq 0$ and $\alpha \neq 0$. We have to show that $P^{\prime}(z)$ has at least one zero in the circle $|z-a+(\alpha / 2)| \leq|\alpha / 2|$. Assume the contrary. That is, assume that all the zeros of $P^{\prime}(z)$ lie in $|z-a+(\alpha / 2)|>|\alpha / 2|$. Since the point $\alpha$ does not lie in $|z-\alpha+(\alpha / 2)|>|\alpha / 2|$, it follows from Lemma 2 that all the zeros of the $(n-2)$ th polar derivative

$$
D_{a}^{n-2} P^{\prime}(z)=D_{a} D_{a} \cdots D_{a} P^{\prime}(z)
$$

of $P^{\prime}(z)$ lie in $|z-\alpha+(\alpha / 2)|>|\alpha / 2|$. But $D_{\alpha}^{n-2} P^{\prime}(z)$ is a polynomial of degree one and its only zero is (see [9, p. 235, Problem V 137]) given by

$$
z=a-\frac{(n-1) P^{\prime}(a)}{P^{\prime \prime}(a)}=a-\frac{(n-1) Q(a)}{2 Q^{\prime}(a)} \text {, }
$$

so that

$$
\frac{1}{a-2}=\frac{2 Q^{\prime}(a)}{(n-1) Q(a)}
$$

This gives with the help of the hypothesis

$$
\operatorname{Re} \frac{\alpha}{a-z}=2 \operatorname{Re} \frac{\alpha Q^{\prime}(a)}{(n-1) Q(a)} \geq 1
$$

which implies

$$
\left|1-\frac{\alpha}{2(\alpha-z)}\right| \leq\left|\frac{\alpha}{2(a-z)}\right| \text {. }
$$

This shows that the only zero of $D_{a}^{n-2} P^{\prime}(z)$ lies in $|z-\alpha+(\alpha / 2)| \leq|\alpha / 2|$, which is a contradiction and therefore the desired result follows.

Proof of Theorem 5. Here we have $P(z)=(z-1) Q(z)$, so that $P^{\prime}(1)=Q(1)$ and $P^{\prime \prime}(1)=2 Q^{\prime}(1)$. Since $Q(z)$ has all its zeros in 
$|z| \geq 1$, therefore, if $z_{1}, z_{2}, \ldots, z_{n-1}$ are the zeros of $Q(z)$, then $\left|z_{j}\right| \geq 1, j=1,2, \ldots, n-1$, and

$$
\frac{z Q^{\prime}(z)}{Q(z)}=\sum_{j=1}^{n-1} \frac{z}{z-z_{j}}
$$

Now for points $z=e^{i \theta}, 0 \leq \theta<2 \pi$, which are not the zeros of $Q(z)$, we have

$$
\operatorname{Re} \frac{e^{i \theta} Q^{\prime}\left(e^{i \theta}\right)}{Q\left(e^{i \theta}\right)}=\sum_{j=1}^{n-1} \frac{e^{i \theta}}{e^{i \theta}-z_{j}} \leq \sum_{j=1}^{n-1} \frac{1}{2}=\frac{n-1}{2}
$$

This implies

$$
\left|e^{i \theta} Q^{\prime}\left(e^{i \theta}\right)\right| \leq\left|(n-1) Q\left(e^{i \theta}\right)-e^{i \theta} Q^{\prime}\left(e^{i \theta}\right)\right|
$$

for points $e^{i \theta}, 0 \leq \theta<2 \pi$, which are not the zeros of $Q(z)$. Since this inequality obviously holds for points $e^{i \theta}$ which are the zeros of $Q(z)$, therefore, it follows that

$$
\left|Q^{\prime}(z)\right| \leq\left|(n-1) Q(z)-z Q^{\prime}(z)\right| \text { for }|z|=1 \text {. }
$$

If $P^{\prime \prime}(1)=0$, then $P^{\prime}(z)$ has at least one zero in $\left|z-\frac{1}{2}\right| \geq \frac{1}{2}$. Because if all the zeros of $P^{\prime}(z)$ lie in $\left|z-\frac{1}{2}\right|<\frac{1}{2}$, then by the Gauss-lucas theorem, all the zeros of $P^{\prime \prime}(z)$ also lie in $\left|z-\frac{1}{2}\right|<\frac{1}{2}$. Since $P^{\prime \prime}(1)=0$ and 1 does not lie in $\left|z-\frac{1}{2}\right|<\frac{1}{2}$, we get a contradiction.

We now suppose that $P^{\prime \prime}(1) \neq 0$, so that $Q^{\prime}(1) \neq 0$. We have to show that $P^{\prime}(z)$ cannot have all its zeros in the disk $\left|z-\frac{1}{2}\right|<\frac{1}{2}$. Assume that all the zeros of $P^{\prime}(z)$ lie in $\left|z-\frac{1}{2}\right|<\frac{1}{2}$. Since 1 does not lie in $\left|z-\frac{1}{2}\right|<\frac{1}{2}$, it follows by Lemma 2 that all the zeros of $(n-2)$ th polar derivative

$$
D_{1}^{n-2} P^{\prime}(z)=D_{1} D_{1} \ldots D_{1} P^{\prime}(z)
$$

of $P^{\prime}(z)$ lie in $\left|z-\frac{1}{2}\right|<\frac{1}{2}$. But the only zero of the polynomial $D_{1}^{n-2} P^{\prime}(z)$, which is of the first degree, is given by (see [9, p. 235])

$$
z=1-\frac{(n-1) P^{\prime}(1)}{P^{\prime \prime}(1)}=1-\frac{(n-1) Q(1)}{2 Q^{\prime}(1)}
$$


This gives, with the help of (6),

$$
\left|z-\frac{1}{2}\right|=\frac{1}{2}\left|\frac{Q^{\prime}(1)-(n-1) Q(1)}{Q^{\prime}(1)}\right| \geq \frac{1}{2} \text {. }
$$

This shows that the only zero of the polynomial $D_{1}^{n-2} P^{\prime}(z)$ lies in $\left|z-\frac{1}{2}\right| \geq \frac{1}{2}$, which is a contradiction and therefore the result follows.

Proof of Theorem 6. Since $|P(z)|$ takes its maximum at $z=e^{i \theta}$ on $|z|=1$, it follows that (see [8, p. 132, Problem III 144])

$e^{i \theta} P^{\prime}\left(e^{i \theta}\right) / P\left(e^{i \theta}\right)$ is real and positive and therefore $P^{\prime}\left(e^{i \theta}\right) \neq 0$. We have to show that $P(z)$ has at least one zero in $\left|z-\left(e^{i \theta} / 2\right)\right| \geq \frac{1}{2}$. Suppose that all the zeros of $P(z)$ lie in $\left|z-\left(e^{i \theta} / 2\right)\right|<\frac{1}{2}$. Since $e^{i \theta}$ does not lie in $\mid z-\left(e^{i \theta} / 2\right)<\frac{1}{2}$, it follows from Lemma 2 that all the zeros of the $(n-1)$ th polar derivative

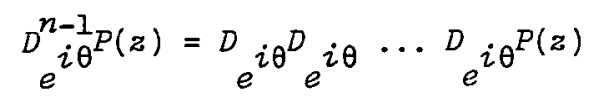

of $P(z)$ lie in $\left|z-\left(e^{i \theta} / 2\right)\right|<\frac{1}{2}$. But $D^{n-1} e^{i \theta} P(z)$ is a polynomial of degree 1 and its only zero (see [9, p. 235, Problem V 137]) is given by

$$
\boldsymbol{z}=e^{i \theta}-\frac{n P\left(e^{i \theta}\right)}{P^{\prime}\left(e^{i \theta}\right)} \text {. }
$$

With the help of Lemma 3 , this zero lies in

$$
\begin{aligned}
\left|z-\frac{e^{i \theta}}{2}\right| & =\left|\frac{e^{i \theta}}{2}-\frac{n P\left(e^{i \theta}\right)}{P^{\prime}\left(e^{i \theta}\right)}\right| \geq \frac{n\left|P\left(e^{i \theta}\right)\right|}{\left|P^{\prime}\left(e^{i \theta}\right)\right|}-\frac{1}{2} \\
& \geq 1-\frac{1}{2}=\frac{1}{2},
\end{aligned}
$$

which is a contradiction and Theorem 6 is established.

\section{References}

[1] Abdul Aziz and Q.G. Mohammad, "Simple proof of a theorem of Erdös and Lax", Proc. Amer. Math. Soc. 80 (1980), 119-122. 
[2] F. Gacs, "On polynomials whose zeros are in the unit disk", J. Math. Anal. App Z. 36 (1971), 627-637.

[3] A.W. Goodman, Q.I. Rahman and J.S. Ratti, "On the zeros of a polynomial and its derivative", Proc. Amer. Math. Soc. 21 (1969), 273-274.

[4] W.K. Hayman, Research problems in function theory (Athlone Press, University of London, London, 1967).

[5] M. Marden, Geometry of polynomials, and edition (Mathematical Surveys, 3. American Mathematical Society, Providence, Rhode Island, 1966).

[6] M. Marden, "Much ado about nothing", Amer. Math. Monthly 83 (1976), 788-798.

[7] A. Meir and A. Sharma, "On Ilyeff's conjecture", Pacific J. Math. 31 (1969), 459-467.

[8] G. Pólyá and G. Szegö, Problems and theorems in analysis (translated by D. Aeppli. Springer-Verlag, Berlin, Heidelberg, New York, 1972).

[9] G. Pólyá and G. Szegö, Problems and theorems in analysis, Volume II (translated by C.E. Billigheimer. Springer-Verlag, New York, Heidelberg, Berlin, 1976).

[10] Z. Rubinstein, "On a problem of Ilyeff", Pacific J. Math. 26 (1968), 158-161.

[11] A.C. Schaeffer, "Inequalities of A. Markoff and S. Bernstein for polynomials and related functions", Bulz. Amer. Math. Soc. 47 (1941), 565-579.

[12] G. Schmeisser, "On Ilieff's conjecture", Math. 2. 156 (1977), $165-173$.

Post-Graduate Department of Mathematics, University of Kashmir, Hazratbal Srinagar - 190006, Kashmir, India. 